\title{
A FUSE SURVEY OF DISK-ACCRETING CATACLYSMIC VARIABLES
}

\author{
C. S. Froning, ${ }^{1}$ K. S. Long, ${ }^{2}$ P. Szkody, ${ }^{3}$ and B. T. Gänsicke ${ }^{4}$
}

We present initial results of a survey of the FUV spectra of disk-accreting cataclysmic variables obtained with the Far Ultraviolet Spectroscopic Explorer (FUSE).

FUSE covers the $905-1188 \AA$ range at spectral resolutions $\simeq 0.1 \AA$. To date, F'USE has observed more than 65 cataclysmic variables (CVs). Publiclyavailable data include observations of 11 dwarf novae (DN), 15 non-magnetic novalikes (NLs), 7 intermediate polars and DQ Her stars, at least 15 polars, and 4 super-soft X-ray binaries. The FUV spectra of CVs are highly complex, with emission from multiple continuum and spectral line source regions. We have instituted an FUV observational survey of C $\mathrm{Vs}$ with FUSE. Combining the survey observations with data from targeted programs, we have created timeaveraged spectra of all publicly-available FUSE spectra of disk-accreting CVs. The survey is on-going; here, we give an initial presentation of the characteristic FUV spectra of disk-accreting CVs.

Figure 1 shows example FUV spectra of two quiescent DN and two NLs. The spectrum of U Gem is populated by absorption lines of HI, HeII, and ionized metals and is dominated by the WD. Several other quiescent DN, including SS Aur, Z Cam, VW Hyi, and WZ Sge, also show WD spectra (e.g., Froning et al. 2001, Long et al. 2003, Godon et al. 2003). This is not true of all DN, however, as the spectrum of EK TrA demonstrates. EK TrA, along with EM Cyg, SS Cyg, RU Peg, and SW UMa, have FUV spectra with relatively flat continua and strong, broad emission lines of OVI, CIII, and NIII. The underlying WD is not visible, and the source of the emission spectrum remains poorly understood.

Also shown in Figure 1 are typical FUV spectra of non-eclipsing NLs. RZ Gru has strong, broad, blueshifted OVI absorption. RX And and SS Cyg in outburst, IX Vel, and RW Sex show similar spec-

\footnotetext{
${ }^{1}$ CASA, University of Colorado, 389 UCB, Boulder, CO 80309 USA (cfroning@casa.colorado.edu).

${ }^{2}$ STSci, 3700 San Martin Dr. , Baltimore, MD 21218 USA(long@stsci.edu).

${ }^{3}$ Astronomy Dept., Univ. of Washington, Seattle, WA 98195 USA(szkody@astro.washington.edu).

${ }^{4}$ Dept. of Physics and Astronomy, Univ. of Southampton, Highfield, Southampton SO17 1BJ UK(btg@astro.soton.ac. uk).
}

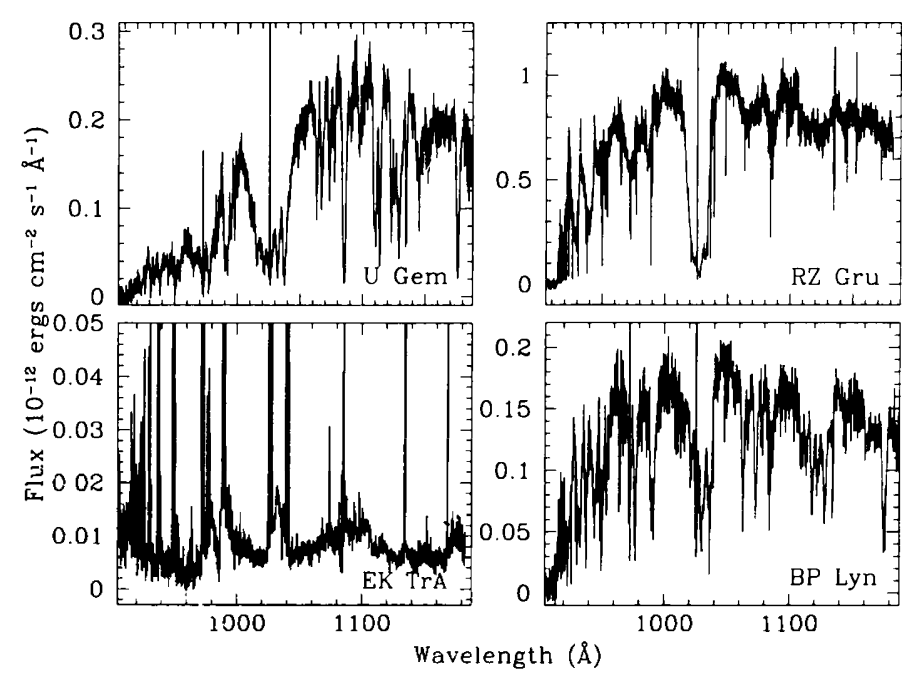

Fig. 1. Example FUV spectra of disk-accreting CVs. The two panels on the left show FUSE spectra of DN in quiescence: $U$ Gem in the upper panel and FK TrA in the lower panel. The two panels on the right show two high-state NLs: RZ Gru in the upper panel and BP Lyn in the lower panel.

tra, which are believed to be dominated by continuum emission from a stcady-state accretion disk and line absorption in a disk wind (e.g., Prinja et al. 2003). In contrast, numerous high state ('Vs. including U Gem in outburst, V795 Her, AH Men. LS Peg, V3885 Sgr, and IX Vel, show spectra similar to that of BP Lyn, with narrow, low-relocity absorption lines that atre unlikely to originate in the inner accretion disk or in a disk wind (Froning et al. 2001). A vertically-extender chromosphere in the outer accretion disk is one possible site for the line absorption. Not shown here are spectra of eclipsing NLs and IPs. The eclipsing systems show strong, broad emission lines or a complex blend of spectral emission and absorption components (e.g.. U.X UM $\mathrm{I}_{\mathrm{a}}$ : Froning et al. 2003). The IPs also have emission spectra. often superimposed on a blue continumm.

\section{REFEREN('ES'}

Froning, C. S., et al. 2003, IpJ, 5\&.1. 133 Froning, C. S., et al. 2001, IpJ, 562. 963 Godon, P., et al. 2003, ApJ, submitted Long, K. S., et al. 2003, ApJ, 5(91, 1172 Prinja. R. K., ct al. 2003, MN, 340.5.51 